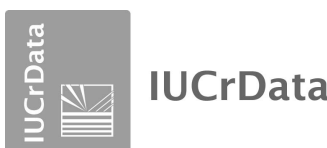

ISSN 2414-3146

Received 28 November 2017

Accepted 20 December 2017

Edited by P. C. Healy, Griffith University, Australia

Keywords: crystal structure; Monoketone analogue; $E$ configuration.

CCDC reference: 1567484

Structural data: full structural data are available from iucrdata.iucr.org

\section{(1E,4E)-1,5-Bis(2,6-dichlorophenyl)penta-1,4-dien- 3-one}

Era Dravida Thendral, ${ }^{a}$ U. Mohamooda Sumaya, ${ }^{b}$ S. Gomathi, ${ }^{c}$ K Biruntha ${ }^{b}$ and G. Usha ${ }^{\mathrm{a} *}$

aPG and Research Department of Physics, Queen Mary's College, Chennai-4, Tamilnadu, India, 'bepartment of Physics, Bharathi Women's College, Chennai-108, Tamilnadu, India, and 'PG Department of Physics, Bhaktavatsalam Memorial College for Women, Chennai-80, Tamilnadu, India. *Correspondence e-mail: guqmc@yahoo.com

The asymmetric unit of the title compound, $\mathrm{C}_{17} \mathrm{H}_{10} \mathrm{Cl}_{4} \mathrm{O}$, consists of one independent molecule and two molecules each located on twofold symmetry axes through the central $\mathrm{C}=\mathrm{O}$ bond such that they each contribute half a molecule each to the asymmetric unit. The dihedral angles between the rings in the three molecules are 73.1 (3), 65.3 (3) and 75.4 (3) $)^{\circ}$. In the crystal, molecules are linked through $\mathrm{C}-\mathrm{H} \cdot \mathrm{O}$. hydrogen bonds, generating undulated molecular sheets lying parallel to (110).
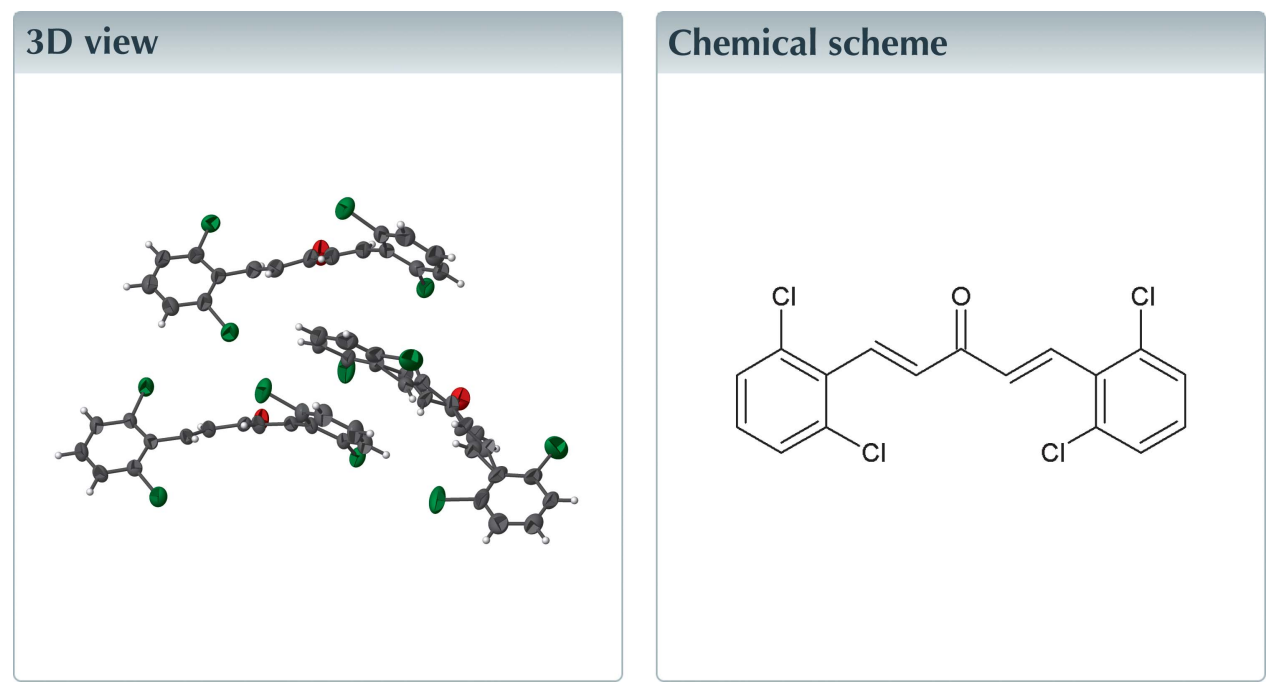

\section{Structure description}

In the asymmetric unit of the title compound (Fig. 1), one independent molecule and two molecules each located on twofold symmetry axes through the central $\mathrm{C}=\mathrm{O}$ bond such that they each contribute half a molecule each to the asymmetric unit. The atoms $\mathrm{C} 33$ and $\mathrm{C} 34$ of the $B$ molecule $(\mathrm{C} 27-\mathrm{C} 35 / \mathrm{Cl} 1 / \mathrm{Cl} 2 / \mathrm{O})$ are disordered over two sets of sites with occupancy factors of $0.471(14)$ and $0.529(14)$. No abnormalities are observed in the bond lengths and angles of the asymmetric unit molecules and are typical of such compounds (Huang et al., 2011).

In the crystal, the molecules are linked primarily via $\mathrm{C}-\mathrm{H} \cdots \mathrm{O}$ hydrogen bonds, forming molecular chains (Table 1 and Fig. 2) with $\mathrm{C}-\mathrm{H} \cdots \mathrm{Cl}$ hydrogen bonds also contributing to the cohesion of the crystal.

\section{Synthesis and crystallization}

The title compound was synthesized by following the published procedure (Lee et al., 2009). A mixture of 2,6 dicholorobenzaldehyde (4.8 g, $0.027 \mathrm{~mol})$ and acetone $(1 \mathrm{ml})$ in 


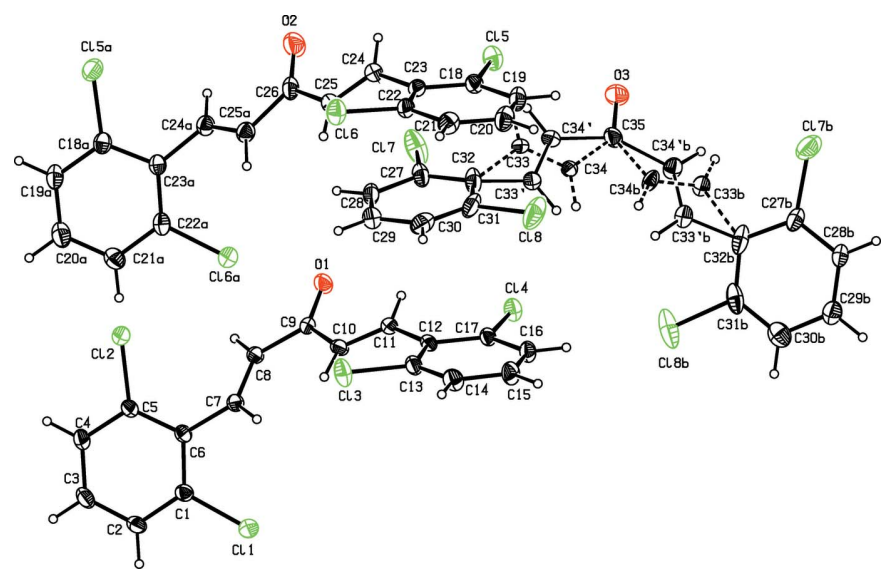

Figure 1

An ORTEP plot of the title compound with three molecules in the asymmetric unit, with displacement ellipsoids drawn at $20 \%$ probability level. Both disorder components are shown. [Symmetry codes: (a) $-x$, $1-y, z ;$ (b) $-x+\frac{1}{2},-y+\frac{3}{2}, z$.]

the presence of $10 \% \mathrm{NaOH}$ along with $50 \mathrm{ml}$ methanol was prepared and the solution was stirred for about $15 \mathrm{~h}$. Ice cubes were added to enhance the precipitation. The resultant precipitate was collected and washed with distilled water to drain excess $\mathrm{NaOH}$ from the product. Single crystals of diffraction quality were grown from chloroform solution by slow evaporation (m.p. $371 \mathrm{~K}$ ).

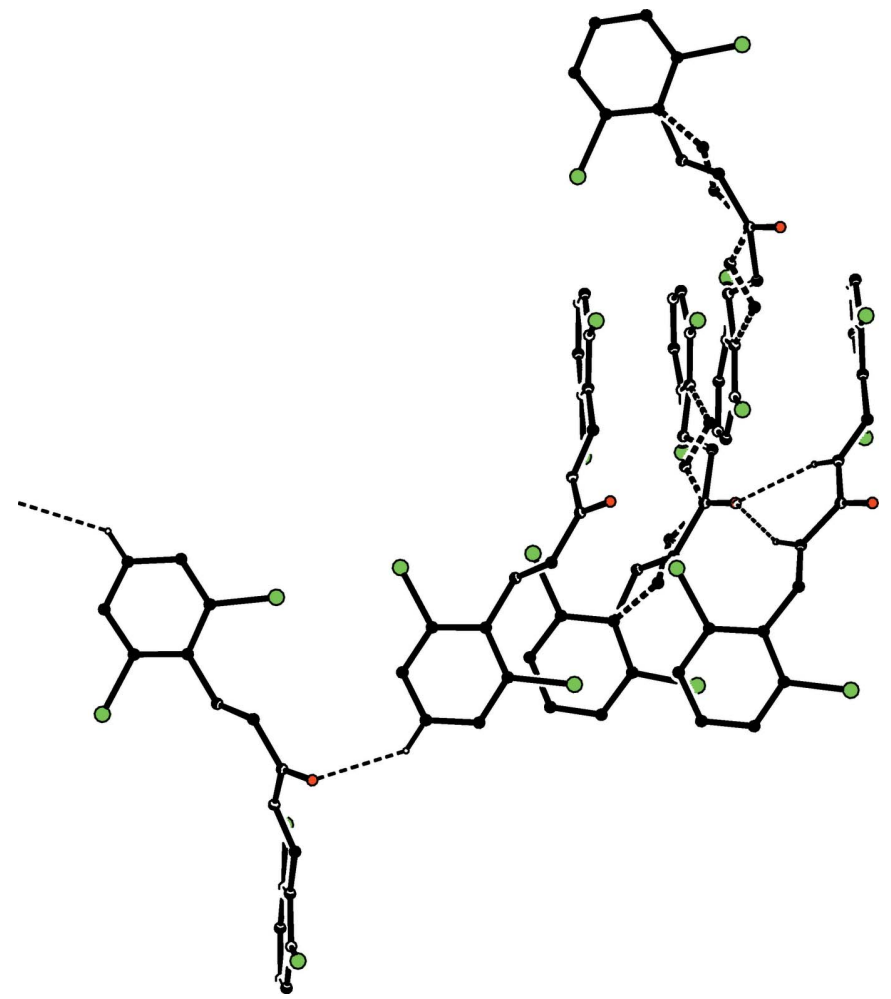

Figure 2

Crystal packing of the title compound. The dashed lines indicate hydrogen bonding. Hydrogen atoms not involved in hydrogen bonding are omitted for clarity.
Table 1

Hydrogen-bond geometry $\left(\AA{ }^{\circ}\right)$.

\begin{tabular}{lllll}
\hline$D-\mathrm{H} \cdots A$ & $D-\mathrm{H}$ & $\mathrm{H} \cdots A$ & $D \cdots A$ & $D-\mathrm{H} \cdots A$ \\
\hline $\mathrm{C} 14-\mathrm{H} 14 \cdots \mathrm{Cl} 4^{\mathrm{i}}$ & 0.93 & 2.86 & $3.750(6)$ & 162 \\
$\mathrm{C} 25-\mathrm{H} 25 \cdots 3^{\mathrm{ii}}$ & 0.93 & 2.57 & $3.444(8)$ & 156 \\
$\mathrm{C} 30-\mathrm{H} 30 \cdots \mathrm{Cl} 7^{\mathrm{i}}$ & 0.93 & 2.90 & $3.784(8)$ & 159
\end{tabular}

Symmetry codes: (i) $-x+\frac{1}{4}, y-\frac{1}{4}, z-\frac{1}{4}$; (ii) $x-\frac{1}{4},-y+\frac{5}{4}, z-\frac{1}{4}$.

Table 2

Experimental details.

\begin{tabular}{|c|c|}
\hline \multicolumn{2}{|l|}{ Crystal data } \\
\hline Chemical formula & $\mathrm{C}_{17} \mathrm{H}_{10} \mathrm{Cl}_{4} \mathrm{O}$ \\
\hline$M_{\mathrm{r}}$ & 372.05 \\
\hline Crystal system, space group & Orthorhombic, $F d d 2$ \\
\hline Temperature $(\mathrm{K})$ & 296 \\
\hline$a, b, c(\AA)$ & $\begin{array}{l}28.1477(8), 17.3385(7), \\
26.1837(10)\end{array}$ \\
\hline$V\left(\AA^{3}\right)$ & $12778.7(8)$ \\
\hline$Z$ & 32 \\
\hline Radiation type & Мо $K \alpha$ \\
\hline$\mu\left(\mathrm{mm}^{-1}\right)$ & 0.74 \\
\hline Crystal size (mm) & $0.20 \times 0.20 \times 0.15$ \\
\hline
\end{tabular}

Data collection

Diffractometer

Absorption correction

$T_{\min }, T_{\max }$

No. of measured, independent and observed $[I>2 \sigma(I)]$ reflections

$R_{\text {int }}$

$(\sin \theta / \lambda)_{\max }\left(\AA^{-1}\right)$

Bruker Kappa APEXII CCD

Multi-scan ( $S A D A B S$; Bruker, 2004)

$0.863,0.895$

$37791,5641,4336$

0.056

0.595

Refinement

$R\left[F^{2}>2 \sigma\left(F^{2}\right)\right], w R\left(F^{2}\right), S \quad 0.040,0.085,1.03$

No. of reflections

5633

No. of parameters

No. of restraints

$\mathrm{H}$-atom treatment

$\Delta \rho_{\max }, \Delta \rho_{\min }\left(\mathrm{e} \AA^{-3}\right)$

Absolute structure

418

64

$\mathrm{H}$-atom parameters constrained

$0.46,-0.47$

Flack $x$ determined using 1755 quotients $\left[\left(I^{+}\right)-\left(I^{-}\right)\right] /\left[\left(I^{+}\right)+\left(I^{-}\right)\right]$

Absolute structure parameter

(Parsons et al., 2013) $-0.01(2)$

Computer programs: APEX2, SAINT and XPREP (Bruker, 2004), SHELXT2014 (Sheldrick, 2015a), SHELXL2014 (Sheldrick, 2015b) and ORTEP-3 for Windows (Farrugia, 2012).

\section{Refinement}

Crystal data, data collection and structure refinement details are summarized in Table 2. Atoms C33 and C34 are disordered over two sets of sites [occupancy ratio 0.471 (14):0.529 (14)].

\section{Acknowledgements}

The authors thank the Central Instrumentation Facility(DSTFIST), Queen Mary's College, Chennai-4 for computing facilities and SAIF, IIT, Madras, for X-ray data collection facilities.

\section{References}

Bruker (2004). APEX2, SAINT, XPREP and $S A D A B S$. Bruker AXS Inc., Madison, Wisconsin, USA.

Farrugia, L. J. (2012). J. Appl. Cryst. 45, 849-854. 
Huang, J.-D., Tang, Q.-Q., Chen, X.-Y., Ye, Y. \& Wang, Y. (2011). Acta Cryst. E67, o758.

Lee, K., Ab Aziz, F. H., Syahida, A., Abas, F., Shaari, K., Israf, D. A. \& Lajis, N. H. (2009). Eur. J. Med. Chem. 44, 3195-3200.
Parsons, S., Flack, H. D. \& Wagner, T. (2013). Acta Cryst. B69, 249259.

Sheldrick, G. M. (2015). Acta Cryst. C71, 3-8. 


\section{full crystallographic data}

IUCrData (2018). 3, x171822 [https://doi.org/10.1107/S2414314617018223]

(1E,4E)-1,5-Bis(2,6-dichlorophenyl)penta-1,4-dien-3-one

Era Dravida Thendral, U. Mohamooda Sumaya, S. Gomathi, K Biruntha and G. Usha

(1E,4E)-1,5-Bis(2,6-dichlorophenyl)penta-1,4-dien-3-one

Crystal data

$\mathrm{C}_{17} \mathrm{H}_{10} \mathrm{Cl}_{4} \mathrm{O}$

$M_{r}=372.05$

Orthorhombic, $F d d 2$

$a=28.1477(8) \AA$

$b=17.3385(7) \AA$

$c=26.1837(10) \AA$

$V=12778.7(8) \AA^{3}$

$Z=32$

$F(000)=6016$

$D_{\mathrm{x}}=1.547 \mathrm{Mg} \mathrm{m}^{-3}$

Melting point: $371 \mathrm{~K}$

Mo $K \alpha$ radiation, $\lambda=0.71073 \AA$

Cell parameters from 6475 reflections

$\theta=2.7-21.9^{\circ}$

$\mu=0.74 \mathrm{~mm}^{-1}$

$T=296 \mathrm{~K}$

Block, yellow

$0.20 \times 0.20 \times 0.15 \mathrm{~mm}$

\section{Data collection}

Bruker Kappa APEXII CCD

diffractometer

Radiation source: fine-focus sealed tube

Graphite monochromator

$\omega$ and $\varphi$ scan

Absorption correction: multi-scan

(SADABS; Bruker, 2004)

$T_{\min }=0.863, T_{\max }=0.895$

\section{Refinement}

Refinement on $F^{2}$

Least-squares matrix: full

$R\left[F^{2}>2 \sigma\left(F^{2}\right)\right]=0.040$

$w R\left(F^{2}\right)=0.085$

$S=1.03$

5633 reflections

418 parameters

64 restraints

Hydrogen site location: inferred from

neighbouring sites
37791 measured reflections

5641 independent reflections

4336 reflections with $I>2 \sigma(I)$

$R_{\text {int }}=0.056$

$\theta_{\text {max }}=25.0^{\circ}, \theta_{\min }=2.1^{\circ}$

$h=-32 \rightarrow 33$

$k=-20 \rightarrow 20$

$l=-31 \rightarrow 31$

$\mathrm{H}$-atom parameters constrained

$w=1 /\left[\sigma^{2}\left(F_{\mathrm{o}}^{2}\right)+(0.0294 P)^{2}+21.0031 P\right]$

where $P=\left(F_{\mathrm{o}}^{2}+2 F_{\mathrm{c}}{ }^{2}\right) / 3$

$(\Delta / \sigma)_{\max }<0.001$

$\Delta \rho_{\max }=0.46 \mathrm{e} \AA^{-3}$

$\Delta \rho_{\min }=-0.47 \mathrm{e} \AA^{-3}$

Absolute structure: Flack $x$ determined using 1755 quotients $\left[\left(I^{+}\right)-\left(I^{-}\right)\right] /\left[\left(I^{+}\right)+\left(I^{-}\right)\right]$(Parsons et al., 2013)

Absolute structure parameter: $-0.01(2)$

\section{Special details}

Geometry. All esds (except the esd in the dihedral angle between two 1.s. planes) are estimated using the full covariance matrix. The cell esds are taken into account individually in the estimation of esds in distances, angles and torsion angles; correlations between esds in cell parameters are only used when they are defined by crystal symmetry. An approximate (isotropic) treatment of cell esds is used for estimating esds involving l.s. planes.

Refinement. $\mathrm{H}$ atoms were positioned geometrically and treated as riding on their parent atoms and refined with, $\mathrm{C}-\mathrm{H}$ distance of $0.93 \AA$. 
Fractional atomic coordinates and isotropic or equivalent isotropic displacement parameters $\left(\AA^{2}\right)$

\begin{tabular}{|c|c|c|c|c|c|}
\hline & $x$ & $y$ & $z$ & $U_{\text {iso }} * / U_{\text {eq }}$ & Occ. $(<1)$ \\
\hline $\mathrm{C} 1$ & $-0.11267(18)$ & $1.0169(3)$ & 0.15237 (19) & 0.0423 (13) & \\
\hline $\mathrm{C} 2$ & $-0.15359(19)$ & $1.0469(3)$ & $0.1311(2)$ & $0.0496(14)$ & \\
\hline $\mathrm{H} 2$ & -0.1518 & 1.0793 & 0.1029 & $0.060 *$ & \\
\hline $\mathrm{C} 3$ & $-0.1968(2)$ & 1.0285 & $0.1519(2)$ & $0.0572(16)$ & \\
\hline H3 & -0.2245 & 1.0489 & 0.1380 & $0.069 *$ & \\
\hline $\mathrm{C} 4$ & $-0.19913(18)$ & $0.9798(3)$ & $0.1935(2)$ & $0.0525(15)$ & \\
\hline $\mathrm{H} 4$ & -0.2284 & 0.9661 & 0.2071 & $0.063^{*}$ & \\
\hline $\mathrm{C} 5$ & $-0.15811(18)$ & $0.9516(3)$ & $0.2146(2)$ & $0.0446(13)$ & \\
\hline C6 & $-0.11302(17)$ & $0.9690(3)$ & $0.19511(19)$ & $0.0389(12)$ & \\
\hline $\mathrm{C} 7$ & $-0.06800(17)$ & 0.9365 & $0.2143(2)$ & $0.0404(12)$ & \\
\hline H7 & -0.0469 & 0.9185 & 0.1896 & $0.049^{*}$ & \\
\hline $\mathrm{C} 8$ & $-0.05401(16)$ & $0.9301(3)$ & $0.2622(2)$ & $0.0407(12)$ & \\
\hline $\mathrm{H} 8$ & -0.0745 & 0.9484 & 0.2873 & $0.049^{*}$ & \\
\hline C9 & $-0.00870(15)$ & 0.8964 & $0.2793(2)$ & $0.0394(12)$ & \\
\hline $\mathrm{C} 10$ & $0.02339(18)$ & 0.8609 & $0.2416(2)$ & $0.0453(13)$ & \\
\hline $\mathrm{H} 10$ & 0.0133 & 0.8548 & 0.2080 & $0.054^{*}$ & \\
\hline C11 & $0.06613(17)$ & 0.8379 & $0.2548(2)$ & $0.0403(12)$ & \\
\hline H11 & 0.0734 & 0.8433 & 0.2893 & $0.048^{*}$ & \\
\hline $\mathrm{C} 12$ & $0.10397(16)$ & 0.8048 & $0.22319(19)$ & $0.0398(12)$ & \\
\hline $\mathrm{C} 13$ & $0.09792(18)$ & $0.7573(3)$ & $0.1799(2)$ & $0.0478(14)$ & \\
\hline $\mathrm{C} 14$ & $0.1352(2)$ & $0.7286(4)$ & $0.1525(2)$ & $0.0584(16)$ & \\
\hline H14 & 0.1297 & 0.6973 & 0.1243 & $0.070^{*}$ & \\
\hline $\mathrm{C} 15$ & 0.18132 (19) & $0.7462(4)$ & $0.1668(2)$ & $0.0590(17)$ & \\
\hline H15 & 0.2068 & 0.7271 & 0.1480 & $0.071 *$ & \\
\hline $\mathrm{C} 16$ & $0.18946(18)$ & 0.7915 & $0.2083(2)$ & $0.0510(14)$ & \\
\hline H16 & 0.2204 & 0.8033 & 0.2179 & $0.061 *$ & \\
\hline $\mathrm{C} 17$ & $0.15191(17)$ & $0.8196(3)$ & $0.23576(19)$ & 0.0403 (12) & \\
\hline $\mathrm{C} 18$ & $0.16222(18)$ & $0.4241(3)$ & $0.3399(2)$ & $0.0491(14)$ & \\
\hline C19 & $0.2023(2)$ & $0.4001(3)$ & $0.3140(3)$ & $0.0608(17)$ & \\
\hline H19 & 0.2322 & 0.4166 & 0.3244 & $0.073 *$ & \\
\hline $\mathrm{C} 20$ & $0.1977(2)$ & $0.3517(4)$ & 0.2729 & 0.0699 (19) & \\
\hline $\mathrm{H} 20$ & 0.2246 & 0.3357 & 0.2551 & $0.084 *$ & \\
\hline $\mathrm{C} 21$ & 0.1535 & $0.3268(4)$ & $0.2580(2)$ & 0.0608 (17) & \\
\hline $\mathrm{H} 21$ & 0.1503 & 0.2934 & 0.2304 & $0.073^{*}$ & \\
\hline $\mathrm{C} 22$ & $0.11380(17)$ & 0.3517 & $0.2843(2)$ & $0.0513(15)$ & \\
\hline $\mathrm{C} 23$ & $0.11649(17)$ & 0.4019 & $0.3262(2)$ & $0.0431(13)$ & \\
\hline $\mathrm{C} 24$ & $0.07580(18)$ & $0.4322(3)$ & $0.3552(2)$ & $0.0490(14)$ & \\
\hline $\mathrm{H} 24$ & 0.0772 & 0.4289 & 0.3907 & $0.059 *$ & \\
\hline $\mathrm{C} 25$ & $0.03839(19)$ & 0.4629 & $0.3354(2)$ & $0.0507(14)$ & \\
\hline $\mathrm{H} 25$ & 0.0351 & 0.4620 & 0.3000 & $0.061 *$ & \\
\hline $\mathrm{C} 26$ & 0.0000 & 0.5000 & $0.3665(4)$ & $0.057(2)$ & \\
\hline $\mathrm{C} 27$ & $0.09628(19)$ & $0.6931(3)$ & $0.3546(2)$ & $0.0588(16)$ & \\
\hline $\mathrm{C} 28$ & $0.05807(18)$ & 0.6714 & 0.3254 & $0.0537(15)$ & \\
\hline $\mathrm{H} 28$ & 0.0275 & 0.6858 & 0.3350 & $0.064^{*}$ & \\
\hline $\mathrm{C} 29$ & 0.0650 & 0.6288 & 0.2826 & $0.0643(17)$ & \\
\hline
\end{tabular}




\begin{tabular}{|c|c|c|c|c|c|}
\hline $\mathrm{H} 29$ & 0.0391 & 0.6139 & 0.2629 & $0.077^{*}$ & \\
\hline $\mathrm{C} 30$ & $0.1100(2)$ & $0.6077(4)$ & $0.2681(3)$ & $0.0722(19)$ & \\
\hline $\mathrm{H} 30$ & 0.1146 & 0.5779 & 0.2390 & $0.087 *$ & \\
\hline $\mathrm{C} 31$ & $0.1477(2)$ & $0.6308(4)$ & $0.2967(3)$ & $0.074(2)$ & \\
\hline C32 & $0.14291(19)$ & $0.6749(4)$ & $0.3408(3)$ & $0.071(2)$ & \\
\hline $\mathrm{C} 33$ & $0.1775(4)$ & $0.6990(8)$ & $0.3877(6)$ & $0.045(4)$ & $0.471(14)$ \\
\hline H33 & 0.1691 & 0.6870 & 0.4211 & $0.054 *$ & $0.471(14)$ \\
\hline C34 & $0.2169(4)$ & $0.7348(8)$ & $0.3785(6)$ & $0.052(4)$ & $0.471(14)$ \\
\hline H34 & 0.2235 & 0.7510 & 0.3454 & $0.063 *$ & $0.471(14)$ \\
\hline C33' & $0.1895(4)$ & $0.7043(6)$ & $0.3532(5)$ & $0.050(3)$ & $0.529(14)$ \\
\hline H33' & 0.2107 & 0.7175 & 0.3274 & $0.060^{*}$ & $0.529(14)$ \\
\hline $\mathrm{C} 34^{\prime}$ & $0.2015(5)$ & $0.7121(9)$ & $0.4010(5)$ & $0.054(4)$ & $0.529(14)$ \\
\hline H34' & 0.1808 & 0.6947 & 0.4261 & $0.064 *$ & $0.529(14)$ \\
\hline $\mathrm{C} 35$ & 0.2500 & 0.7500 & $0.4180(3)$ & $0.056(2)$ & \\
\hline $\mathrm{O} 1$ & $0.00129(11)$ & $0.8985(2)$ & $0.32475(14)$ & $0.0559(10)$ & \\
\hline $\mathrm{O} 2$ & 0.0000 & 0.5000 & $0.4127(3)$ & $0.081(2)$ & \\
\hline $\mathrm{O} 3$ & 0.2500 & 0.7500 & $0.4629(2)$ & $0.0734(18)$ & \\
\hline $\mathrm{Cl1}$ & $-0.05851(5)$ & $1.04252(9)$ & $0.12522(6)$ & $0.0594(4)$ & \\
\hline $\mathrm{Cl} 2$ & $-0.16449(5)$ & $0.88981(11)$ & $0.26605(7)$ & $0.0744(5)$ & \\
\hline $\mathrm{Cl} 3$ & $0.04160(5)$ & $0.72921(11)$ & $0.16049(7)$ & $0.0731(5)$ & \\
\hline Cl4 & $0.16511(5)$ & $0.87707(9)$ & $0.28818(6)$ & $0.0567(4)$ & \\
\hline $\mathrm{Cl} 5$ & $0.16975(6)$ & $0.48515(9)$ & $0.39223(6)$ & $0.0666(5)$ & \\
\hline $\mathrm{Cl} 6$ & $0.05927(5)$ & $0.31438(10)$ & $0.26448(6)$ & $0.0669(4)$ & \\
\hline $\mathrm{Cl} 7$ & $0.08458(7)$ & $0.74969(13)$ & $0.40754(9)$ & $0.1053(8)$ & \\
\hline $\mathrm{Cl} 8$ & $0.20421(6)$ & $0.60238(12)$ & $0.27670(11)$ & $0.1188(10)$ & \\
\hline
\end{tabular}

Atomic displacement parameters $\left(\AA^{2}\right)$

\begin{tabular}{lllllll}
\hline & $U^{11}$ & $U^{22}$ & $U^{33}$ & $U^{12}$ & $U^{13}$ & $U^{23}$ \\
\hline $\mathrm{C} 1$ & $0.045(3)$ & $0.048(3)$ & $0.034(3)$ & $0.001(3)$ & $-0.002(2)$ & $-0.001(3)$ \\
$\mathrm{C} 2$ & $0.052(3)$ & $0.052(3)$ & $0.045(4)$ & $0.007(3)$ & $-0.007(3)$ & $0.010(3)$ \\
$\mathrm{C} 3$ & $0.046(3)$ & $0.061(4)$ & $0.065(4)$ & $0.007(3)$ & $-0.019(3)$ & $0.007(3)$ \\
$\mathrm{C} 4$ & $0.035(3)$ & $0.059(4)$ & $0.064(4)$ & $0.005(3)$ & $-0.005(3)$ & $0.006(3)$ \\
$\mathrm{C} 5$ & $0.039(3)$ & $0.045(3)$ & $0.050(3)$ & $0.003(2)$ & $-0.007(2)$ & $0.009(3)$ \\
$\mathrm{C} 6$ & $0.038(3)$ & $0.038(3)$ & $0.040(3)$ & $0.005(2)$ & $-0.003(2)$ & $-0.002(2)$ \\
$\mathrm{C} 7$ & $0.036(3)$ & $0.042(3)$ & $0.043(3)$ & $0.008(2)$ & $0.003(2)$ & $0.003(3)$ \\
$\mathrm{C} 8$ & $0.036(3)$ & $0.051(3)$ & $0.035(3)$ & $0.007(2)$ & $0.004(2)$ & $0.002(3)$ \\
$\mathrm{C} 9$ & $0.032(3)$ & $0.047(3)$ & $0.039(3)$ & $-0.004(2)$ & $0.002(2)$ & $0.004(3)$ \\
$\mathrm{C} 10$ & $0.041(3)$ & $0.061(4)$ & $0.034(3)$ & $0.012(3)$ & $0.001(2)$ & $0.008(3)$ \\
$\mathrm{C} 11$ & $0.037(3)$ & $0.043(3)$ & $0.040(3)$ & $0.002(2)$ & $0.000(2)$ & $0.005(2)$ \\
$\mathrm{C} 12$ & $0.035(3)$ & $0.045(3)$ & $0.039(3)$ & $0.007(2)$ & $-0.004(2)$ & $0.005(3)$ \\
$\mathrm{C} 13$ & $0.037(3)$ & $0.058(4)$ & $0.048(4)$ & $0.005(3)$ & $-0.005(2)$ & $0.001(3)$ \\
$\mathrm{C} 14$ & $0.056(4)$ & $0.075(4)$ & $0.044(4)$ & $0.007(3)$ & $-0.002(3)$ & $-0.013(3)$ \\
$\mathrm{C} 15$ & $0.038(3)$ & $0.078(4)$ & $0.061(4)$ & $0.015(3)$ & $0.007(3)$ & $-0.010(4)$ \\
$\mathrm{C} 16$ & $0.034(3)$ & $0.062(4)$ & $0.057(4)$ & $0.004(3)$ & $0.000(3)$ & $-0.003(3)$ \\
$\mathrm{C} 17$ & $0.033(3)$ & $0.042(3)$ & $0.046(3)$ & $0.005(2)$ & $-0.002(2)$ & $0.001(3)$ \\
$\mathrm{C} 18$ & $0.043(3)$ & $0.047(3)$ & $0.058(4)$ & $0.000(3)$ & $-0.003(3)$ & $0.007(3)$ \\
$\mathrm{C} 19$ & $0.036(3)$ & $0.055(4)$ & $0.091(5)$ & $0.000(3)$ & $0.001(3)$ & $0.003(4)$
\end{tabular}




$\begin{array}{lllllll} & & & & & \\ \text { C20 } & 0.048(4) & 0.066(4) & 0.096(6) & 0.003(3) & 0.011(4) & 0.000(4) \\ \text { C21 } & 0.060(4) & 0.057(4) & 0.066(4) & 0.007(3) & 0.010(3) & -0.003(3) \\ \text { C22 } & 0.038(3) & 0.054(3) & 0.062(4) & 0.003(3) & 0.001(3) & 0.010(3) \\ \text { C23 } & 0.039(3) & 0.042(3) & 0.049(3) & 0.000(2) & 0.002(2) & 0.008(3) \\ \text { C24 } & 0.043(3) & 0.055(4) & 0.048(4) & -0.001(3) & -0.002(3) & 0.006(3) \\ \text { C25 } & 0.045(3) & 0.059(4) & 0.049(4) & -0.001(3) & -0.005(3) & 0.004(3) \\ \text { C26 } & 0.034(4) & 0.063(6) & 0.074(7) & 0.001(4) & 0.000 & 0.000 \\ \text { C27 } & 0.041(3) & 0.052(4) & 0.083(5) & -0.005(3) & -0.016(3) & -0.002(3) \\ \text { C28 } & 0.034(3) & 0.056(4) & 0.070(4) & -0.005(3) & -0.004(3) & 0.003(3) \\ \text { C29 } & 0.051(4) & 0.074(5) & 0.067(5) & -0.005(3) & -0.006(3) & 0.007(4) \\ \text { C30 } & 0.076(5) & 0.064(4) & 0.077(5) & -0.002(4) & 0.019(4) & 0.013(4) \\ \text { C31 } & 0.042(4) & 0.057(4) & 0.123(7) & -0.001(3) & 0.024(4) & 0.028(4) \\ \text { C32 } & 0.037(3) & 0.047(4) & 0.129(6) & -0.007(3) & -0.016(4) & 0.014(4) \\ \text { C33 } & 0.042(7) & 0.060(7) & 0.034(8) & -0.008(6) & -0.003(6) & 0.015(7) \\ \text { C34 } & 0.042(7) & 0.080(9) & 0.034(8) & -0.022(6) & -0.012(6) & 0.014(7) \\ \text { C33' } & 0.043(7) & 0.049(6) & 0.058(8) & -0.003(5) & -0.004(6) & 0.013(6) \\ \text { C34' } & 0.050(9) & 0.071(8) & 0.040(8) & -0.008(7) & -0.007(7) & 0.013(6) \\ \text { C35 } & 0.042(5) & 0.083(6) & 0.042(5) & 0.012(4) & 0.000 & 0.000 \\ \text { O1 } & 0.044(2) & 0.087(3) & 0.038(2) & 0.0066(19) & -0.0018(17) & -0.001(2) \\ \text { O2 } & 0.060(4) & 0.118(6) & 0.066(5) & 0.016(4) & 0.000 & 0.000 \\ \text { O3 } & 0.074(4) & 0.106(5) & 0.040(4) & -0.001(3) & 0.000 & 0.000 \\ \text { C11 } & 0.0509(8) & 0.0797(11) & 0.0476(9) & -0.0002(7) & 0.0032(7) & 0.0106(8) \\ \text { C12 } & 0.0428(8) & 0.0948(13) & 0.0857(12) & -0.0048(8) & -0.0023(8) & 0.0453(11) \\ \text { C13 } & 0.0445(9) & 0.0937(12) & 0.0811(12) & 0.0003(8) & -0.0166(8) & -0.0252(10) \\ \text { C14 } & 0.0433(8) & 0.0685(10) & 0.0581(9) & -0.0003(7) & -0.0047(7) & -0.0120(8) \\ \text { C15 } & 0.0561(9) & 0.0670(10) & 0.0768(12) & -0.0040(7) & -0.0114(8) & -0.0088(9) \\ \text { C16 } & 0.0511(8) & 0.0788(11) & 0.0707(11) & -0.0064(8) & -0.0010(8) & -0.0184(9) \\ \text { C17 } & 0.0755(14) & 0.1122(16) & 0.1282(18) & 0.0179(11) & -0.0433(12) & -0.0494(14) \\ \text { C18 } & 0.0568(10) & 0.0889(15) & 0.211(3) & 0.0153(10) & 0.0501(14) & 0.0386(17)\end{array}$

Geometric parameters $(\AA, \stackrel{\circ}{)})$

\begin{tabular}{llll}
\hline $\mathrm{C} 1-\mathrm{C} 2$ & $1.381(7)$ & $\mathrm{C} 20-\mathrm{C} 21$ & $1.374(8)$ \\
$\mathrm{C} 1-\mathrm{C} 6$ & $1.394(7)$ & $\mathrm{C} 20-\mathrm{H} 20$ & 0.9300 \\
$\mathrm{C} 1-\mathrm{C} 11$ & $1.740(5)$ & $\mathrm{C} 21-\mathrm{C} 22$ & 0.9300 \\
$\mathrm{C} 2-\mathrm{C} 3$ & $1.370(8)$ & $\mathrm{C} 21-\mathrm{H} 21$ & $1.402(8)$ \\
$\mathrm{C} 2-\mathrm{H} 2$ & 0.9300 & $\mathrm{C} 22-\mathrm{C} 23$ & $1.745(5)$ \\
$\mathrm{C} 3-\mathrm{C} 4$ & $1.380(8)$ & $\mathrm{C} 22-\mathrm{C} 16$ & $1.472(7)$ \\
$\mathrm{C} 3-\mathrm{H} 3$ & 0.9300 & $\mathrm{C} 23-\mathrm{C} 24$ & $1.290(7)$ \\
$\mathrm{C} 4-\mathrm{C} 5$ & $1.370(7)$ & $\mathrm{C} 24-\mathrm{C} 25$ & 0.9300 \\
$\mathrm{C} 4-\mathrm{H} 4$ & 0.9300 & $\mathrm{C} 24-\mathrm{H} 24$ & $1.498(8)$ \\
$\mathrm{C} 5-\mathrm{C} 6$ & $1.401(7)$ & $\mathrm{C} 25-\mathrm{C} 26$ & 0.9300 \\
$\mathrm{C} 5-\mathrm{C} 2$ & $1.731(5)$ & $\mathrm{C} 25-\mathrm{H} 25$ & $1.211(10)$ \\
$\mathrm{C} 6-\mathrm{C} 7$ & $\mathrm{C} 26-\mathrm{O} 2$ & $1.498(8)$ \\
$\mathrm{C} 7-\mathrm{C} 8$ & $1.474(7)$ & $\mathrm{C} 26-\mathrm{C} 25^{\mathrm{i}}$ & $1.371(7)$ \\
$\mathrm{C} 7-\mathrm{H} 7$ & $1.320(7)$ & $\mathrm{C} 27-\mathrm{C} 28$ & $1.397(8)$ \\
$\mathrm{C} 8-\mathrm{C} 9$ & 0.9300 & $\mathrm{C} 27-\mathrm{C} 32$ & $1.731(7)$ \\
$\mathrm{C} 8-\mathrm{H} 8$ & $1.473(7)$ & $\mathrm{C} 27-\mathrm{C} 17$ &
\end{tabular}




\begin{tabular}{|c|c|c|c|}
\hline $\mathrm{C} 9-\mathrm{O} 1$ & $1.224(6)$ & $\mathrm{C} 28-\mathrm{C} 29$ & $1.357(8)$ \\
\hline $\mathrm{C} 9-\mathrm{C} 10$ & $1.473(7)$ & $\mathrm{C} 28-\mathrm{H} 28$ & 0.9300 \\
\hline $\mathrm{C} 10-\mathrm{C} 11$ & $1.314(7)$ & $\mathrm{C} 29-\mathrm{C} 30$ & $1.371(8)$ \\
\hline $\mathrm{C} 10-\mathrm{H} 10$ & 0.9300 & $\mathrm{C} 29-\mathrm{H} 29$ & 0.9300 \\
\hline $\mathrm{C} 11-\mathrm{C} 12$ & $1.466(7)$ & $\mathrm{C} 30-\mathrm{C} 31$ & $1.360(9)$ \\
\hline $\mathrm{C} 11-\mathrm{H} 11$ & 0.9300 & $\mathrm{C} 30-\mathrm{H} 30$ & 0.9300 \\
\hline $\mathrm{C} 12-\mathrm{C} 13$ & $1.411(7)$ & $\mathrm{C} 31-\mathrm{C} 32$ & $1.393(10)$ \\
\hline $\mathrm{C} 12-\mathrm{C} 17$ & $1.413(7)$ & $\mathrm{C} 31-\mathrm{Cl} 8$ & $1.744(6)$ \\
\hline $\mathrm{C} 13-\mathrm{C} 14$ & $1.367(7)$ & $\mathrm{C} 32-\mathrm{C} 33^{\prime}$ & $1.444(11)$ \\
\hline $\mathrm{C} 13-\mathrm{Cl} 3$ & $1.735(5)$ & $\mathrm{C} 32-\mathrm{C} 33$ & $1.622(15)$ \\
\hline $\mathrm{C} 14-\mathrm{C} 15$ & $1.385(8)$ & $\mathrm{C} 33-\mathrm{C} 34$ & $1.293(16)$ \\
\hline C14-H14 & 0.9300 & C33-H33 & 0.9300 \\
\hline $\mathrm{C} 15-\mathrm{C} 16$ & $1.360(8)$ & $\mathrm{C} 34-\mathrm{C} 35$ & $1.418(12)$ \\
\hline $\mathrm{C} 15-\mathrm{H} 15$ & 0.9300 & $\mathrm{C} 34-\mathrm{C} 34^{\mathrm{ii}}$ & $1.94(2)$ \\
\hline $\mathrm{C} 16-\mathrm{C} 17$ & $1.368(7)$ & C34-H34 & 0.9300 \\
\hline $\mathrm{C} 16-\mathrm{H} 16$ & 0.9300 & $\mathrm{C} 33^{\prime}-\mathrm{C} 34^{\prime}$ & $1.303(16)$ \\
\hline $\mathrm{C} 17-\mathrm{Cl} 4$ & $1.736(5)$ & $\mathrm{C} 33^{\prime}-\mathrm{H} 33^{\prime}$ & 0.9300 \\
\hline $\mathrm{C} 18-\mathrm{C} 19$ & $1.380(8)$ & $\mathrm{C} 34^{\prime}-\mathrm{C} 35$ & $1.578(13)$ \\
\hline $\mathrm{C} 18-\mathrm{C} 23$ & $1.391(7)$ & $\mathrm{C} 34^{\prime}-\mathrm{H} 34^{\prime}$ & 0.9300 \\
\hline $\mathrm{C} 18-\mathrm{Cl} 5$ & $1.744(6)$ & $\mathrm{C} 35-\mathrm{O} 3$ & $1.177(9)$ \\
\hline $\mathrm{C} 19-\mathrm{C} 20$ & $1.371(9)$ & $\mathrm{C} 35-\mathrm{C} 34^{\mathrm{ii}}$ & $1.418(12)$ \\
\hline C19-H19 & 0.9300 & $\mathrm{C} 35-\mathrm{C} 34^{\prime i \mathrm{ii}}$ & $1.578(13)$ \\
\hline $\mathrm{C} 2-\mathrm{C} 1-\mathrm{C} 6$ & $122.8(5)$ & $\mathrm{C} 20-\mathrm{C} 21-\mathrm{C} 22$ & $119.5(6)$ \\
\hline $\mathrm{C} 2-\mathrm{C} 1-\mathrm{C} 11$ & $118.0(4)$ & $\mathrm{C} 20-\mathrm{C} 21-\mathrm{H} 21$ & 120.3 \\
\hline $\mathrm{C} 6-\mathrm{C} 1-\mathrm{Cl} 1$ & $119.1(4)$ & $\mathrm{C} 22-\mathrm{C} 21-\mathrm{H} 21$ & 120.3 \\
\hline $\mathrm{C} 3-\mathrm{C} 2-\mathrm{C} 1$ & $119.6(5)$ & $\mathrm{C} 21-\mathrm{C} 22-\mathrm{C} 23$ & $122.8(5)$ \\
\hline $\mathrm{C} 3-\mathrm{C} 2-\mathrm{H} 2$ & 120.2 & $\mathrm{C} 21-\mathrm{C} 22-\mathrm{C} 16$ & $116.5(5)$ \\
\hline $\mathrm{C} 1-\mathrm{C} 2-\mathrm{H} 2$ & 120.2 & $\mathrm{C} 23-\mathrm{C} 22-\mathrm{C} 16$ & $120.7(4)$ \\
\hline $\mathrm{C} 2-\mathrm{C} 3-\mathrm{C} 4$ & $119.8(5)$ & $\mathrm{C} 18-\mathrm{C} 23-\mathrm{C} 22$ & $115.1(5)$ \\
\hline $\mathrm{C} 2-\mathrm{C} 3-\mathrm{H} 3$ & 120.1 & $\mathrm{C} 18-\mathrm{C} 23-\mathrm{C} 24$ & $119.2(5)$ \\
\hline $\mathrm{C} 4-\mathrm{C} 3-\mathrm{H} 3$ & 120.1 & $\mathrm{C} 22-\mathrm{C} 23-\mathrm{C} 24$ & $125.7(5)$ \\
\hline $\mathrm{C} 5-\mathrm{C} 4-\mathrm{C} 3$ & $119.8(5)$ & $\mathrm{C} 25-\mathrm{C} 24-\mathrm{C} 23$ & $125.1(5)$ \\
\hline $\mathrm{C} 5-\mathrm{C} 4-\mathrm{H} 4$ & 120.1 & $\mathrm{C} 25-\mathrm{C} 24-\mathrm{H} 24$ & 117.5 \\
\hline $\mathrm{C} 3-\mathrm{C} 4-\mathrm{H} 4$ & 120.1 & $\mathrm{C} 23-\mathrm{C} 24-\mathrm{H} 24$ & 117.5 \\
\hline $\mathrm{C} 4-\mathrm{C} 5-\mathrm{C} 6$ & $122.7(5)$ & $\mathrm{C} 24-\mathrm{C} 25-\mathrm{C} 26$ & $123.1(6)$ \\
\hline $\mathrm{C} 4-\mathrm{C} 5-\mathrm{Cl} 2$ & $116.6(4)$ & $\mathrm{C} 24-\mathrm{C} 25-\mathrm{H} 25$ & 118.4 \\
\hline $\mathrm{C} 6-\mathrm{C} 5-\mathrm{Cl} 2$ & $120.7(4)$ & $\mathrm{C} 26-\mathrm{C} 25-\mathrm{H} 25$ & 118.4 \\
\hline $\mathrm{C} 1-\mathrm{C} 6-\mathrm{C} 5$ & $115.3(4)$ & $\mathrm{O} 2-\mathrm{C} 26-\mathrm{C} 25$ & $122.9(4)$ \\
\hline $\mathrm{C} 1-\mathrm{C} 6-\mathrm{C} 7$ & $119.6(5)$ & $\mathrm{O} 2-\mathrm{C} 26-\mathrm{C} 25^{\mathrm{i}}$ & $122.9(4)$ \\
\hline $\mathrm{C} 5-\mathrm{C} 6-\mathrm{C} 7$ & $125.0(5)$ & $\mathrm{C} 25-\mathrm{C} 26-\mathrm{C} 25^{\mathrm{i}}$ & $114.1(8)$ \\
\hline $\mathrm{C} 8-\mathrm{C} 7-\mathrm{C} 6$ & $127.8(5)$ & $\mathrm{C} 28-\mathrm{C} 27-\mathrm{C} 32$ & $122.1(6)$ \\
\hline $\mathrm{C} 8-\mathrm{C} 7-\mathrm{H} 7$ & 116.1 & $\mathrm{C} 28-\mathrm{C} 27-\mathrm{Cl} 7$ & $116.9(5)$ \\
\hline $\mathrm{C} 6-\mathrm{C} 7-\mathrm{H} 7$ & 116.1 & $\mathrm{C} 32-\mathrm{C} 27-\mathrm{Cl} 7$ & $120.9(5)$ \\
\hline $\mathrm{C} 7-\mathrm{C} 8-\mathrm{C} 9$ & $125.5(5)$ & $\mathrm{C} 29-\mathrm{C} 28-\mathrm{C} 27$ & $119.7(5)$ \\
\hline $\mathrm{C} 7-\mathrm{C} 8-\mathrm{H} 8$ & 117.2 & $\mathrm{C} 29-\mathrm{C} 28-\mathrm{H} 28$ & 120.1 \\
\hline $\mathrm{C} 9-\mathrm{C} 8-\mathrm{H} 8$ & 117.2 & $\mathrm{C} 27-\mathrm{C} 28-\mathrm{H} 28$ & 120.1 \\
\hline $\mathrm{O} 1-\mathrm{C} 9-\mathrm{C} 8$ & $118.9(4)$ & $\mathrm{C} 28-\mathrm{C} 29-\mathrm{C} 30$ & $120.5(6)$ \\
\hline
\end{tabular}


$\mathrm{O} 1-\mathrm{C} 9-\mathrm{C} 10$

$\mathrm{C} 8-\mathrm{C} 9-\mathrm{C} 10$

$\mathrm{C} 11-\mathrm{C} 10-\mathrm{C} 9$

$\mathrm{C} 11-\mathrm{C} 10-\mathrm{H} 10$

C9- $\mathrm{C} 10-\mathrm{H} 10$

$\mathrm{C} 10-\mathrm{C} 11-\mathrm{C} 12$

C10-C11-H11

C12-C11-H11

$\mathrm{C} 13-\mathrm{C} 12-\mathrm{C} 17$

$\mathrm{C} 13-\mathrm{C} 12-\mathrm{C} 11$

$\mathrm{C} 17-\mathrm{C} 12-\mathrm{C} 11$

$\mathrm{C} 14-\mathrm{C} 13-\mathrm{C} 12$

$\mathrm{C} 14-\mathrm{C} 13-\mathrm{Cl} 3$

$\mathrm{C} 12-\mathrm{C} 13-\mathrm{Cl} 3$

C13-C14-C15

C13-C14-H14

C15-C14-H14

C16-C15-C14

C16-C15-H15

$\mathrm{C} 14-\mathrm{C} 15-\mathrm{H} 15$

C15-C16-C17

C15-C16-H16

C17-C16-H16

$\mathrm{C} 16-\mathrm{C} 17-\mathrm{C} 12$

$\mathrm{C} 16-\mathrm{C} 17-\mathrm{Cl} 4$

$\mathrm{C} 12-\mathrm{C} 17-\mathrm{Cl} 4$

$\mathrm{C} 19-\mathrm{C} 18-\mathrm{C} 23$

$\mathrm{C} 19-\mathrm{C} 18-\mathrm{Cl} 5$

$\mathrm{C} 23-\mathrm{C} 18-\mathrm{Cl} 5$

$\mathrm{C} 20-\mathrm{C} 19-\mathrm{C} 18$

C20-C19-H19

C18-C19-H19

$\mathrm{C} 19-\mathrm{C} 20-\mathrm{C} 21$

$\mathrm{C} 19-\mathrm{C} 20-\mathrm{H} 20$

$\mathrm{C} 21-\mathrm{C} 20-\mathrm{H} 20$

$\mathrm{C} 6-\mathrm{C} 1-\mathrm{C} 2-\mathrm{C} 3$

$\mathrm{C} 11-\mathrm{C} 1-\mathrm{C} 2-\mathrm{C} 3$

$\mathrm{C} 1-\mathrm{C} 2-\mathrm{C} 3-\mathrm{C} 4$

$\mathrm{C} 2-\mathrm{C} 3-\mathrm{C} 4-\mathrm{C} 5$

$\mathrm{C} 3-\mathrm{C} 4-\mathrm{C} 5-\mathrm{C} 6$

$\mathrm{C} 3-\mathrm{C} 4-\mathrm{C} 5-\mathrm{Cl} 2$

$\mathrm{C} 2-\mathrm{C} 1-\mathrm{C} 6-\mathrm{C} 5$

$\mathrm{C} 11-\mathrm{C} 1-\mathrm{C} 6-\mathrm{C} 5$

$\mathrm{C} 2-\mathrm{C} 1-\mathrm{C} 6-\mathrm{C} 7$

$\mathrm{C} 11-\mathrm{C} 1-\mathrm{C} 6-\mathrm{C} 7$

$\mathrm{C} 4-\mathrm{C} 5-\mathrm{C} 6-\mathrm{C} 1$

$\mathrm{Cl} 2-\mathrm{C} 5-\mathrm{C} 6-\mathrm{C} 1$
$121.6(4)$

$119.5(5)$

120.7 (5)

119.6

119.6

$129.4(5)$

115.3

115.3

$114.1(4)$

$126.4(5)$

$119.4(5)$

$122.8(5)$

116.5 (4)

120.7 (4)

119.8 (5)

120.1

120.1

$120.1(5)$

119.9

119.9

119.7 (5)

120.1

120.1

$123.4(5)$

$117.0(4)$

$119.5(4)$

$123.0(5)$

$118.1(4)$

$118.9(4)$

$119.7(5)$

120.2

120.2

$120.0(6)$

120.0

120.0

$-1.0(8)$

$-179.3(4)$

$-0.6(9)$

1.7 (9)

$-1.3(9)$

$-179.1(4)$

$1.4(8)$

179.7 (4)

$177.4(5)$

$-4.3(7)$

$-0.3(8)$

$177.5(4)$
$\mathrm{C} 28-\mathrm{C} 29-\mathrm{H} 29$

C30-C29- 229

$\mathrm{C} 31-\mathrm{C} 30-\mathrm{C} 29$

$\mathrm{C} 31-\mathrm{C} 30-\mathrm{H} 30$

$\mathrm{C} 29-\mathrm{C} 30-\mathrm{H} 30$

$\mathrm{C} 30-\mathrm{C} 31-\mathrm{C} 32$

$\mathrm{C} 30-\mathrm{C} 31-\mathrm{Cl} 8$

$\mathrm{C} 32-\mathrm{C} 31-\mathrm{Cl} 8$

$\mathrm{C} 31-\mathrm{C} 32-\mathrm{C} 27$

C31-C32-C33'

C27-C $32-\mathrm{C} 33^{\prime}$

$\mathrm{C} 31-\mathrm{C} 32-\mathrm{C} 33$

$\mathrm{C} 27-\mathrm{C} 32-\mathrm{C} 33$

C34-C33-C32

C34-C33-H33

C $32-\mathrm{C} 33-\mathrm{H} 33$

$\mathrm{C} 33-\mathrm{C} 34-\mathrm{C} 35$

$\mathrm{C} 33-\mathrm{C} 34-\mathrm{C} 34^{\mathrm{ii}}$

$\mathrm{C} 35-\mathrm{C} 34-\mathrm{C} 34^{\mathrm{ii}}$

C33-C $34-\mathrm{H} 34$

$\mathrm{C} 35-\mathrm{C} 34-\mathrm{H} 34$

C34 - C34-H34

C34'-C $33^{\prime}-\mathrm{C} 32$

$\mathrm{C} 34^{\prime}-\mathrm{C} 33^{\prime}-\mathrm{H} 33^{\prime}$

C $32-\mathrm{C} 33^{\prime}-\mathrm{H} 33^{\prime}$

C $33^{\prime}-\mathrm{C} 34^{\prime}-\mathrm{C} 35$

$\mathrm{C} 33^{\prime}-\mathrm{C} 34^{\prime}-\mathrm{H} 34^{\prime}$

C $35-\mathrm{C} 34^{\prime}-\mathrm{H} 34^{\prime}$

$\mathrm{O} 3-\mathrm{C} 35-\mathrm{C} 34^{\mathrm{ii}}$

$\mathrm{O} 3-\mathrm{C} 35-\mathrm{C} 34$

$\mathrm{C} 34^{\mathrm{ii}}-\mathrm{C} 35-\mathrm{C} 34$

$\mathrm{O} 3-\mathrm{C} 35-\mathrm{C} 34^{\prime}$

$\mathrm{O} 3-\mathrm{C} 35-\mathrm{C} 34^{\prime i \mathrm{ii}}$

C $34^{\prime}-\mathrm{C} 35-\mathrm{C} 34^{\prime \prime i}$

$\mathrm{C} 19-\mathrm{C} 18-\mathrm{C} 23-\mathrm{C} 22$

$\mathrm{C} 15-\mathrm{C} 18-\mathrm{C} 23-\mathrm{C} 22$

$\mathrm{C} 19-\mathrm{C} 18-\mathrm{C} 23-\mathrm{C} 24$

$\mathrm{C} 15-\mathrm{C} 18-\mathrm{C} 23-\mathrm{C} 24$

$\mathrm{C} 21-\mathrm{C} 22-\mathrm{C} 23-\mathrm{C} 18$

$\mathrm{C} 16-\mathrm{C} 22-\mathrm{C} 23-\mathrm{C} 18$

$\mathrm{C} 21-\mathrm{C} 22-\mathrm{C} 23-\mathrm{C} 24$

$\mathrm{C} 16-\mathrm{C} 22-\mathrm{C} 23-\mathrm{C} 24$

$\mathrm{C} 18-\mathrm{C} 23-\mathrm{C} 24-\mathrm{C} 25$

$\mathrm{C} 22-\mathrm{C} 23-\mathrm{C} 24-\mathrm{C} 25$

$\mathrm{C} 23-\mathrm{C} 24-\mathrm{C} 25-\mathrm{C} 26$

$\mathrm{C} 24-\mathrm{C} 25-\mathrm{C} 26-\mathrm{O} 2$
119.8

119.8

$119.3(7)$

120.3

120.3

$122.8(6)$

$117.6(7)$

$119.5(6)$

$115.4(6)$

$107.0(8)$

$135.7(8)$

$135.3(7)$

$108.1(8)$

$119.8(12)$

120.1

120.1

$121.1(13)$

162.7 (14)

46.9 (6)

119.4

119.4

74.0

$119.3(13)$

120.3

120.3

$122.6(13)$

118.7

118.7

$136.9(6)$

$136.9(6)$

86.3 (12)

106.4 (6)

106.4 (6)

$147.3(12)$

$1.2(8)$

-178.9 (4)

$-178.3(5)$

$1.6(7)$

$-0.9(8)$

176.5 (4)

$178.5(5)$

$-4.1(8)$

130.7 (6)

-48.8 (9)

-173.8 (4)

-4.3 (6) 


$\begin{array}{ll}\mathrm{C} 4-\mathrm{C} 5-\mathrm{C} 6-\mathrm{C} 7 & -176.0(5) \\ \mathrm{C} 12-\mathrm{C} 5-\mathrm{C} 6-\mathrm{C} 7 & 1.7(8) \\ \mathrm{C} 1-\mathrm{C} 6-\mathrm{C} 7-\mathrm{C} 8 & 137.8(6) \\ \mathrm{C} 5-\mathrm{C} 6-\mathrm{C} 7-\mathrm{C} 8 & -46.6(9) \\ \mathrm{C} 6-\mathrm{C} 7-\mathrm{C} 8-\mathrm{C} 9 & 179.3(5) \\ \mathrm{C} 7-\mathrm{C} 8-\mathrm{C} 9-\mathrm{O} 1 & 175.2(5) \\ \mathrm{C} 7-\mathrm{C} 8-\mathrm{C} 9-\mathrm{C} 10 & -4.9(8) \\ \mathrm{O} 1-\mathrm{C} 9-\mathrm{C} 10-\mathrm{C} 11 & -7.4(8) \\ \mathrm{C} 8-\mathrm{C} 9-\mathrm{C} 10-\mathrm{C} 11 & 172.7(5) \\ \mathrm{C} 9-\mathrm{C} 10-\mathrm{C} 11-\mathrm{C} 12 & -177.0(5) \\ \mathrm{C} 10-\mathrm{C} 11-\mathrm{C} 12-\mathrm{C} 13 & -32.9(9) \\ \mathrm{C} 10-\mathrm{C} 11-\mathrm{C} 12-\mathrm{C} 17 & 147.6(6) \\ \mathrm{C} 17-\mathrm{C} 12-\mathrm{C} 13-\mathrm{C} 14 & -0.3(8) \\ \mathrm{C} 11-\mathrm{C} 12-\mathrm{C} 13-\mathrm{C} 14 & -179.9(5) \\ \mathrm{C} 17-\mathrm{C} 12-\mathrm{C} 13-\mathrm{C} 13 & 177.0(4) \\ \mathrm{C} 11-\mathrm{C} 12-\mathrm{C} 13-\mathrm{C} 13 & -2.5(7) \\ \mathrm{C} 12-\mathrm{C} 13-\mathrm{C} 14-\mathrm{C} 15 & -0.3(9) \\ \mathrm{C} 13-\mathrm{C} 13-\mathrm{C} 14-\mathrm{C} 15 & -177.7(5) \\ \mathrm{C} 13-\mathrm{C} 14-\mathrm{C} 15-\mathrm{C} 16 & 0.5(9) \\ \mathrm{C} 14-\mathrm{C} 15-\mathrm{C} 16-\mathrm{C} 17 & -0.1(9) \\ \mathrm{C} 15-\mathrm{C} 16-\mathrm{C} 17-\mathrm{C} 12 & -0.6(9) \\ \mathrm{C} 15-\mathrm{C} 16-\mathrm{C} 17-\mathrm{C} 14 & -179.9(5) \\ \mathrm{C} 13-\mathrm{C} 12-\mathrm{C} 17-\mathrm{C} 16 & 0.8(7) \\ \mathrm{C} 11-\mathrm{C} 12-\mathrm{C} 17-\mathrm{C} 16 & -179.7(5) \\ \mathrm{C} 13-\mathrm{C} 12-\mathrm{C} 17-\mathrm{C} 14 & -179.9(4) \\ \mathrm{C} 11-\mathrm{C} 12-\mathrm{C} 17-\mathrm{C} 14 & -0.3(7) \\ \mathrm{C} 23-\mathrm{C} 18-\mathrm{C} 19-\mathrm{C} 20 & -0.5(9) \\ \mathrm{C} 15-\mathrm{C} 18-\mathrm{C} 19-\mathrm{C} 20 & 179.6(5) \\ \mathrm{C} 18-\mathrm{C} 19-\mathrm{C} 20-\mathrm{C} 21 & -0.6(10) \\ \mathrm{C} 19-\mathrm{C} 20-\mathrm{C} 21-\mathrm{C} 22 & 0.9(10) \\ \mathrm{C} 20-\mathrm{C} 21-\mathrm{C} 22-\mathrm{C} 23 & -0.1(9) \\ \mathrm{C} 20-\mathrm{C} 21-\mathrm{C} 22-\mathrm{C} 16 & -177.6(5) \\ & \end{array}$

$\mathrm{C} 24-\mathrm{C} 25-\mathrm{C} 26-\mathrm{C} 25^{\mathrm{i}}$

C $32-\mathrm{C} 27-\mathrm{C} 28-\mathrm{C} 29$

$\mathrm{C} 17-\mathrm{C} 27-\mathrm{C} 28-\mathrm{C} 29$

$\mathrm{C} 27-\mathrm{C} 28-\mathrm{C} 29-\mathrm{C} 30$

$\mathrm{C} 28-\mathrm{C} 29-\mathrm{C} 30-\mathrm{C} 31$

$\mathrm{C} 29-\mathrm{C} 30-\mathrm{C} 31-\mathrm{C} 32$

$\mathrm{C} 29-\mathrm{C} 30-\mathrm{C} 31-\mathrm{Cl} 8$

$\mathrm{C} 30-\mathrm{C} 31-\mathrm{C} 32-\mathrm{C} 27$

$\mathrm{C} 18-\mathrm{C} 31-\mathrm{C} 32-\mathrm{C} 27$

$\mathrm{C} 30-\mathrm{C} 31-\mathrm{C} 32-\mathrm{C} 33^{\prime}$

$\mathrm{C} 18-\mathrm{C} 31-\mathrm{C} 32-\mathrm{C} 33^{\prime}$

$\mathrm{C} 30-\mathrm{C} 31-\mathrm{C} 32-\mathrm{C} 33$

$\mathrm{C} 18-\mathrm{C} 31-\mathrm{C} 32-\mathrm{C} 33$

$\mathrm{C} 28-\mathrm{C} 27-\mathrm{C} 32-\mathrm{C} 31$

$\mathrm{C} 17-\mathrm{C} 27-\mathrm{C} 32-\mathrm{C} 31$

$\mathrm{C} 28-\mathrm{C} 27-\mathrm{C} 32-\mathrm{C} 33^{\prime}$

$\mathrm{C} 17-\mathrm{C} 27-\mathrm{C} 32-\mathrm{C} 33^{\prime}$

$\mathrm{C} 28-\mathrm{C} 27-\mathrm{C} 32-\mathrm{C} 33$

$\mathrm{C} 17-\mathrm{C} 27-\mathrm{C} 32-\mathrm{C} 33$

C31-C32-C33-C34

$\mathrm{C} 27-\mathrm{C} 32-\mathrm{C} 33-\mathrm{C} 34$

$\mathrm{C} 32-\mathrm{C} 33-\mathrm{C} 34-\mathrm{C} 35$

$\mathrm{C} 32-\mathrm{C} 33-\mathrm{C} 34-\mathrm{C} 34^{\mathrm{ii}}$

$\mathrm{C} 31-\mathrm{C} 32-\mathrm{C} 33^{\prime}-\mathrm{C} 34^{\prime}$

$\mathrm{C} 27-\mathrm{C} 32-\mathrm{C} 33^{\prime}-\mathrm{C} 34^{\prime}$

$\mathrm{C} 32-\mathrm{C} 33^{\prime}-\mathrm{C} 34^{\prime}-\mathrm{C} 35$

$\mathrm{C} 33-\mathrm{C} 34-\mathrm{C} 35-\mathrm{O} 3$

$\mathrm{C} 34$ ii- $34-\mathrm{C} 35-\mathrm{O} 3$

$\mathrm{C} 33-\mathrm{C} 34-\mathrm{C} 35-\mathrm{C} 34^{\mathrm{ii}}$

$\mathrm{C} 33^{\prime}-\mathrm{C} 34^{\prime}-\mathrm{C} 35-\mathrm{O} 3$

$\mathrm{C} 33^{\prime}-\mathrm{C} 34^{\prime}-\mathrm{C} 35-\mathrm{C} 34^{\prime \text { ii }}$
$175.7(6)$

$2.1(9)$

$178.1(5)$

$-0.4(10)$

$-0.9(10)$

$0.5(10)$

$179.9(5)$

$1.2(10)$

$-178.3(5)$

$-165.8(7)$

$14.8(8)$

$167.2(9)$

$-12.2(12)$

$-2.5(9)$

$-178.3(5)$

$159.5(9)$

$-16.3(13)$

$-172.2(7)$

$12.0(8)$

$57.6(17)$

$-135.7(13)$

$-173.8(9)$

$-132(4)$

$-145.2(11)$

$51.7(16)$

$-174.9(9)$

$-15.6(19)$

$179.999(1)$

164.4 (19)

$178.7(11)$

$-1.3(11)$

Symmetry codes: (i) $-x,-y+1, z$; (ii) $-x+1 / 2,-y+3 / 2, z$.

Hydrogen-bond geometry $\left(\AA,{ }^{\circ}\right)$

\begin{tabular}{lllll}
\hline$D-\mathrm{H} \cdots A$ & $D-\mathrm{H}$ & $\mathrm{H} \cdots A$ & $D \cdots A$ & $D-\mathrm{H} \cdots A$ \\
\hline $\mathrm{C} 10-\mathrm{H} 10 \cdots \mathrm{Cl} 3$ & 0.93 & 2.63 & $3.160(6)$ & 117 \\
$\mathrm{C} 14-\mathrm{H} 14 \cdots \mathrm{Cl} 4^{\text {iii }}$ & 0.93 & 2.86 & $3.750(6)$ & 162 \\
$\mathrm{C} 25-\mathrm{H} 25 \cdots 3^{\text {iv }}$ & 0.93 & 2.57 & $3.444(8)$ & 156 \\
$\mathrm{C} 30-\mathrm{H} 30 \cdots \mathrm{Cl} 7^{\mathrm{iii}}$ & 0.93 & 2.90 & $3.784(8)$ & 159 \\
$\mathrm{C} 34-\mathrm{H} 34^{\prime} \cdots \mathrm{Cl} 7$ & 0.93 & 2.91 & $3.361(14)$ & 111 \\
\hline
\end{tabular}

Symmetry codes: (iii) $-x+1 / 4, y-1 / 4, z-1 / 4$; (iv) $x-1 / 4,-y+5 / 4, z-1 / 4$. 